\title{
Qualidade de vida em indivíduos com apneia obstrutiva do sono moderada a grave antes e após tratamento com pressão positiva contínua nas vias aéreas
}

\author{
Quality of life in moderate to severe obstructive sleep apnea individuals \\ before and after continuous positive airway pressure treatment \\ Paulo Roberto Ramos Alves' ${ }^{1}$, Fernando Arruda Ramos ${ }^{2,3}$, Thaise Brighente Volpato ${ }^{1}$ \\ ${ }^{1}$ Curso de Medicina, Universidade do Planalto Catarinense (UNIPLAC) - Lages (SC), Brasil. \\ ${ }^{2}$ UNIPLAC - Lages (SC), Brasil. \\ ${ }^{3}$ Departamento de Otorrinolaringologia, Instituto Catarinense do Sono (ICS) - Lages (SC), Brasil.
}

DOI: http://dx.doi.org/10.7322/abcshs.v41i3.903

\begin{abstract}
RESUMO
Introdução: A apneia obstrutiva do sono (AOS) é caracterizada por episódios recorrentes de obstrução parcial ou total das vias aéreas superiores com duração de pelo menos dezsegundos e frequênciamaior que cinco episódios por hora de sono. Objetivo: Analisar a qualidade de vida dos indivíduos portadores de AOS após tratamento com pressão positiva contínua nas vias aéreas (CPAP). Métodos: Estudo realizado com 20 indivíduos portadores de AOS com índice de apneia/hipopneia (IAH) maior que 15, identificados por meio de polissonografia (PSG). Os indivíduos foram submetidos a dois questionários de qualidade de vida antes e depois do tratamento. Resultados: Participaram do estudo 20 indivíduos adultos com idade média de $57 \pm 10$ anos, índice de massa corporal (IMC) médio de $31,6 \pm 4,5 \mathrm{~kg} / \mathrm{m}^{2}$ e circunferência cervical (CC) média de $38,7 \pm 2,8 \mathrm{~cm}$. Observamos queda média de 6,6 $\pm 4,6$ pontos na escala de Epworth e 6,6 61,5 pontos na escala de Stanford, bem como ganhos que variaram de 5 a 100 pontos na escala de qualidade de vida. Conclusão: Indivíduos com AOS moderada a grave obtêm melhora na qualidade de vida com o uso da CPAP.
\end{abstract}

Palavras-chave: apneia do sono tipo obstrutiva; sono; qualidade de vida.

\begin{abstract}
Introduction: Obstructive sleep apnea (OSA) is characterized by recurrent episodes of partial or total obstruction of the upper airway, lasting at least ten seconds each in a frequency greater than five episodes per hour during sleep. Objective: To analyze the quality of life of individuals with OSA after treatment with continuous positive airway pressure (CPAP). Methods: Twenty individuals with OSA and apnea-hypopnea index (AHI) greater than 15 , identified by polysomnography (PSG), were submitted to two quality of life questionnaires before and after CPAP treatment. Results: Twenty subjects completed the protocol. Mean age was $57 \pm 10$ years, mean body mass index (BMI) was $31.6 \pm 4.5 \mathrm{~kg} / \mathrm{m} 2$ and neck circumference (NC) average was $38.7 \pm 2.8 \mathrm{~cm}$. We noted an average loss of $6.6 \pm 4.6$ points in the Epworth scale and $6.6 \pm 1.5$ points on the Stanford scale, besides gains ranging from 5 to 100 points on the scale of quality of life. Conclusion: CPAP improves quality of life in moderate to severe OSA individuals.
\end{abstract}

Keywords: sleep apnea, obstructive; sleep; quality of life. 


\section{INTRODUÇÃO}

O sono é um processo neural absolutamente necessário à homeostase e tem sido definido como um estado fisiológico complexo que requer integração cerebral completa, durante a qual ocorrem alterações dos processos fisiológicos e comportamentais ${ }^{1}$. A apneia obstrutiva do sono (AOS) é um distúrbio muito frequente da respiração no sono, de etiologia multifatorial e ainda não completamente esclarecida. É caracterizada por episódios recorrentes de obstrução parcial ou total das vias aéreas superiores. Na AOS ocorre redução ou cessação completa do fluxo de ar, com pelo menos dez segundos de duração e frequência maior que cinco episódios por hora de sono ${ }^{2}$.

Sintomas como hipersonolência diurna, cansaço, indisposição, cefaleia matinal, alteração cognitiva e depressão são queixas comuns que devem servir de alerta para o possível diagnóstico de apneias obstrutivas ${ }^{3}$.

A AOS afeta $32,9 \%$ dos homens e das mulheres, trazendo consequências bastante negativas para a saúde, segundo recente estudo realizado na cidade de São Paulo ${ }^{4}$. Entre as consequências da AOS estão as doenças cardiovasculares, que incluem alterações no sistema nervoso autônomo, acidente vascular cerebral, hipertensão arterial sistêmica, insuficiência cardíaca esquerda, infarto do miocárdio, arritmias e hipertensão pulmonar, podendo culminar com morte súbita ${ }^{5}$.

Os eventos respiratórios durante a noite, evidenciados na AOS, podem ser do tipo:

1. apneia obstrutiva: caracterizado pela obstrução completa das vias aéreas superiores. Aqui, observamos redução $\geq 90 \%$ do fluxo de ar durante a respiração por pelo menos dez segundos, apesar dos esforços respiratórios contínuos;

2. hipopneia obstrutiva: caracterizado pela redução do fluxo de ar $\geq 30 \%$ do fluxo aéreo normal, com queda de pelo menos $3 \%$ da saturação de oxigênio ${ }^{6}$.

Esses eventos podem ter sua frequência aumentada de acordo com índice de massa corporal (IMC) elevado e aumento da circunferência cervical (CC) $)^{2}$. A polissonografia (PSG) de noite inteira constitui o método diagnóstico padrão ouro para a avaliação dos distúrbios respiratórios do sono. Recomenda-se um registro de pelo menos seis horas para classificar a apneia como: normal $(\mathrm{IAH} \leq 5)$, leve $(\mathrm{IAH}>5 \mathrm{e}$ $\mathrm{IAH} \leq 15)$, moderada $(\mathrm{IAH}>15$ e $\mathrm{IAH} \leq 30)$ e grave $(\mathrm{IAH}>30)^{7}$.

O tratamento mais conservador e eficiente para a AOS moderada a grave é a aplicação noturna de pressão positiva contínua nas vias aéreas (CPAP). A CPAP elimina os eventos de apneia por causa de um suporte pneumático na via aérea que impede o fechamento ou a diminuição do espaço para a passagem de $\operatorname{ar}^{8,9}$.

O presente estudo tem por objetivo comparar a qualidade de vida de indivíduos com AOS moderada a grave antes e após o uso de CPAP.

\section{MÉTODOS}

Trata-se de estudo quantitativo realizado com pacientes de um consultório particular de otorrinolaringologia estabelecido na cidade de Lages (SC). Os indivíduos foram consecutivamente convidados a participar da pesquisa, sem distinção de sexo nem de idade, no período entre janeiro e dezembro de 2014. Aqueles que concordaram em participar do estudo assinaram um termo de consentimento livre e esclarecido (TCLE) e foram submetidos a anamnese detalhada para a obtenção dos dados apresentados na Tabela 1.

Todos os participantes foram submetidos a PSG de noite inteira com mínimo de seis horas. O critério de inclusão na pesquisa foi $A O S \geq 15$ eventos por hora de sono. Indivíduos que não contemplaram o critério de inclusão foram excluídos da pesquisa. Para a coleta de dados, utilizaram-se dois questionários, um com a versão brasileira do Medical Outcomes Study 36, Short-Form Health Survey (SF 36), e outro feito com a junção das escolas de Stanford e Epworth ${ }^{10,11}$. Ambos os instrumentos foram aplicados antes e depois de um período de três meses de tratamento com CPAP durante o sono noturno. Não houve desistências no decorrer do tratamento, e todos os indivíduos foram avaliados após o mesmo intervalo de tempo.

O presente trabalho foi aprovado pelo Comitê de Ética em Pesquisa da Universidade do Planalto Catarinense.

A qualidade de vida dos indivíduos foi avaliada por meio do questionário SF 36, cujos resultados foram analisados em oito domínios, especificados na Tabela 2, com a média de cada variável e seu desvio padrão. Os domínios utilizados foram: capacidade funcional (CF), limitação por aspectos físicos (LAF), dor (D), estado geral de saúde (EGS), vitalidade (V), aspectos sociais (AS), aspectos emocionais (AE) e saúde mental (SM). Os domínios apresentados pelo questionário foram analisados pela raw scale e receberam notas de 0 a 100 , sendo o primeiro a pior e o último a melhor nota; os valores finais não representam nenhuma unidade de medida. As regras para o cálculo das variáveis são reportadas pelo próprio questionário SF 36.

A segunda parte do questionário foi elaborada com os dados de identificação dos indivíduos (IMC, idade e CC) associados às escalas de Stanford e Epworth, com as médias e os desvios padrão de cada escala. A escala de Stanford visou quantificar a intensidade do ronco de 0 a 10, sendo o mínimo a ausência de ronco e a nota máxima relacionada à impossibilidade de o casal dormir no mesmo quarto por conta do ronco. A escala de Epworth quantifica o grau de sonolência diurna, sendo a nota mínima de zero relacionada à sua ausência e aquela acima de 10 pontos considerada sonolência diurna excessiva. Para a análise dos dados de tabulação do SF 36, foram usadas as regras de cálculo impostas pelo próprio questionário, e, para as variáveis de identificação e

Tabela 1: Características dos indivíduos analisados

\begin{tabular}{|l|c|}
\hline Variável & Amostra \\
\hline Indivíduos & 20 pessoas \\
\hline Homens & $50 \%$ \\
\hline Mulheres & $50 \%$ \\
\hline Idade & $57 \pm 10$ anos \\
\hline IMC & $31,6 \pm 4,5 \mathrm{~kg} / \mathrm{m}^{2}$ \\
\hline CC & $38,7 \pm 2,8 \mathrm{~cm}$ \\
\hline
\end{tabular}

IMC: índice de massa corporal; CC: circunferência cervical. 
Tabela 2: Variação da intensidade de ronco, sonolência diurna, índice de apneia e domínios do SF-36 após tratamento com pressão positiva contínua nas vias aéreas (CPAP)

\begin{tabular}{|c|c|c|c|}
\hline Variável & Antes do tratamento & Após o tratamento & $\mathbf{p}$ \\
\hline Escala de Stanford & $7,95 \pm 1,23$ & $1,35 \pm 1,22$ & $3,45 \times 10^{-11}$ \\
\hline Escala de Epworth & $9,95 \pm 2,72$ & $3,30 \pm 1,34$ & $5,41 \times 10^{-12}$ \\
\hline $\mathrm{AlH}$ & $26 \pm 5,54$ & $4,66 \pm 1,54$ & $3,96 \times 10^{-11}$ \\
\hline CF & $33 \pm 19,89$ & $77,50 \pm 14,37$ & $6,98 \times 10^{-9}$ \\
\hline LAF & $35 \pm 31,83$ & $100 \pm 0$ & $2,22 \times 10^{-8}$ \\
\hline $\mathrm{D}$ & $40,60 \pm 13,18$ & $78,55 \pm 14,15$ & $1,73 \times 10^{-12}$ \\
\hline EGS & $48,75 \pm 11,45$ & $69,80 \pm 8,91$ & $1,37 \times 10^{-7}$ \\
\hline V & $44,50 \pm 12$ & $79,12 \pm 7,49$ & $1,44 \times 10^{-10}$ \\
\hline AS & $55,37 \pm 14,91$ & $81,60 \pm 16,07$ & $7,56 \times 10^{-6}$ \\
\hline $\mathrm{AE}$ & $41,86 \pm 41,67$ & $93,54 \pm 19,89$ & $3,06 \times 10^{-5}$ \\
\hline SM & $48,80 \pm 12,82$ & $76,40 \pm 12,64$ & $4,32 \times 10^{-7}$ \\
\hline
\end{tabular}

AlH: índice de apneia por hora de sono; CF: capacidade funcional; LAF: limitação por aspectos físicos; D: dor; EGS: estado geral de saúde; V: vitalidade; AS: aspectos sociais; AE: aspectos emocionais; SM: saúde mental.

cálculo do grau de significância pelo método de t pareado, empregou-se o programa Statistical Package for Social Science (SPSS) versão 20.0.

\section{RESULTADOS}

Foram avaliados 20 indivíduos, dos quais $50 \%$ eram do sexo masculino. A faixa etária da amostra teve média de $57 \pm 10$ anos.

Sobre os achados antropométricos expostos na Tabela 1, observou-se média de IMC de $31,6 \pm 4,5 \mathrm{~kg} / \mathrm{m}^{2}$ e de CC de $38,7 \pm 2,8 \mathrm{~cm}$. Os resultados encontrados com a aplicação do questionário SF 36 foram expostos na Tabela 2, de acordo com os domínios preestabelecidos pelo questionário.

Avaliando-se o quesito CF, $100 \%$ dos indivíduos obtiveram ganho de no mínimo 25 pontos e de no máximo 100 pontos ao final do cálculo do questionário. No domínio LAF, $85 \%$ dos pacientes apresentaram ganhos de no mínimo 50 pontos, $10 \%$ se mantiveram com a mesma pontuação e $5 \%$ tiveram ganho de 25 pontos. O domínio D teve $95 \%$ dos indivíduos com acréscimo de no mínimo 22 pontos na escala, enquanto $5 \%$ permaneceram com pontuação inalterada.

No quarto domínio (EGS), 95\% dos indivíduos obtiveram acréscimo de no mínimo cinco pontos e $5 \%$ permaneceram sem alteração. No quinto domínio $(\mathrm{V})$, todos apresentaram acréscimo de no mínimo 15 pontos em seus índices. No domínio AS, 90\% das pessoas obtiveram acréscimo de 12,5 pontos na escala, $5 \%$ permaneceram com pontuação inalterada e $5 \%$ obtiveram queda de 20 pontos. No domínio AE, $65 \%$ dos indivíduos somaram no mínimo 33 pontos em relação a sua pontuação inicial e 35\% permaneceram com pontuação inalterada. No último domínio analisado (SM), 10\% dos indivíduos mantiveram a mesma pontuação e $90 \%$ obtiveram aumento mínimo de 12 pontos na escala.

$\mathrm{Na}$ escala de Stanford, verificou-se queda nos índices para todos os indivíduos: 95\% apresentaram evolução mínima de quatro pontos e apenas $5 \%$ obtiveram evolução de dois pontos. Na escala de Epworth, 100\% dos indivíduos apresentaram queda de pelo menos dois pontos no índice de sonolência diurna.

\section{DISCUSSÃO}

Os dados antropométricos de CC e IMC vinculados à pesquisa e expressos na Tabela 1 predizem ampla relação de predisposição e piora do quadro de apneia do sono, segundo a literatura. A CC, sozinha, tem sensibilidade de 60,6\% (IC95\% 54,6-66,6\%) e especificidade de $93,4 \%$ (IC95\% 86,3-100\%) como fator preditivo de gravidade da $\mathrm{AOS}^{12}$. Já o IMC, quando evidenciado acima de $32,2 \mathrm{~kg} /$ $\mathrm{m}^{2}$, apresenta sensibilidade de 92,5\% (IC95\% 89,3-95,8\%) e especificidade de 73,9\% (IC95\% 61,2-86,6\%) para diagnóstico e maior gravidade da $\mathrm{AOS}^{13,14}$. Na população estudada em nossa pesquisa, tanto o IMC quanto a CC se mostraram fatores preditivos de gravidade da AOS e, consequentemente, de menor qualidade de vida, conforme pudemos constatar nas avaliações iniciais (Tabela 2).

A escala de Stanford mostrou que, antes do tratamento, os indivíduos tinham um nível de ronco mínimo de cinco pontos, capaz de acordar o companheiro de quarto, fazendo-os, assim, procurar auxílio para tratamento. Após o uso da CPAP, o índice de Stanford caiu em média $6,6 \pm 1,5$ pontos, o que refletiu diretamente na percepção do indivíduo e de seu companheiro (Tabela 2).

Ao analisar os resultados da escala de Epworth, verificou-se que todos os indivíduos apresentavam índices maiores que 5 pontos, dos quais 11 acumulavam no mínimo 10 pontos, demonstrando sonolência diurna excessiva, fato que pode estar relacionado com AOS, haja vista sua queda após o tratamento (Tabela 2). A literatura corrobora os achados que mostram que os indivíduos com grau de AOS moderado a grave, com pontuação igual ou superior a oito na escala de Epworth, terão melhoria dos sintomas de sonolência diurna, do tempo de reação aos estímulos e também da qualidade de vida com o tratamento dos distúrbios respiratórios do sono ${ }^{15}$.

De acordo com Mediano et al. ${ }^{16}$, a AOS impõe uma limitação significativa à qualidade de vida do indivíduo, influenciando negativamente desde tarefas mais rotineiras até casos mais preocupantes, como o aumento do risco de acidentes ocupacionais e automobilísticos, em função da hipersonolência diurna enfrentada por tais indivíduos. Após o uso da CPAP, os índices de Epworth 
diminuíram, alcançando no máximo sete pontos conforme a escala de avaliação, o que não caracteriza sonolência diurna patológica.

O impacto do uso de CPAP na qualidade de vida dos indivíduos foi avaliado de forma subjetiva pelo questionário SF 36 , que indica o grau de percepção do indivíduo sobre sua condição antes e depois do tratamento. Esses dados mostram que houve considerável melhora na qualidade de vida dos indivíduos analisados, comprovando os benefícios do tratamento com CPAP. Existe uma ampla gama de vantagens já comprovadas do uso da CPAP em pessoas com AOS moderada a grave, como redução no índice de apneia/hipopneia (IAH), melhora na arquitetura do sono, melhora da função neurocognitiva, diminuição da pressão arterial sistêmica diurna e noturna e redução da atividade simpática diurna, que repercutirão na qualidade de vida ${ }^{17}$.
Os parâmetros encontrados na aplicação do questionário após o tratamento estão vinculados aos benefícios do uso de CPAP relacionados à eliminação das apneias, ao aumento da saturação da oxi-hemoglobina e à diminuição dos despertares decorrentes de eventos respiratórios, ocorrendo, assim, redução da sonolência diurna excessiva e melhora das funções neuropsíquicas, do desempenho subjetivo no trabalho, dos sintomas relacionados à depressão e da qualidade de vida ${ }^{16-18}$.

Concluímos que os dados obtidos no presente estudo comprovaram que, antes do tratamento, os indivíduos portadores de AOS em graus variados sofriam de algum tipo de prejuízo em suas funções vitais. Após o tratamento com CPAP, evidenciaram-se redução na sonolência diurna e melhora na qualidade de vida dos sujeitos, comprovando o benefício do tratamento da AOS para os indivíduos que participaram da presente pesquisa.

\section{REFERÊNCIAS}

1. Lorenzi Filho G. Apneia obstrutiva do sono: um grave problema de saúde pública. Pneumol Paulista. 2008;21(3):5.

2. Chaves Junior CM, Dal-Fabbro C, Bruin VMS, Tufik S, Bittencourt LRA. Consenso brasileiro de ronco e apneia do sono: aspectos de interesse aos ortodontistas. Dental Press J Orthod. 2011;16(1):34.e1-10. http://dx.doi.org/10.1590/S2176-94512011000100007

3. Campostrini DDA, Prado LBF, Prado GF. Síndrome da apneia obstrutiva do sono e doenças cardiovasculares. Rev Neurocienc. 2014;22(1):102-12.

http://dx.doi.org/10.4181/RNC.2014.22.930.11p

4. Tufik S, Santos-Silva R, Taddei JA, Bittencourt LR. Obstructive sleep apnea syndrome in the São Paulo Epidemiologic Sleep Study. Sleep Med. 2010;11(5):441-6. http://dx.doi.org/10.1016/j.sleep.2009.10.005

5. Cintra FD, Poyares D, Guilleminault C, Carvalho AC, Tufik S, Paola AAV. Alterações cardiovasculares na síndrome da apneia obstrutiva do sono. Arq Bras Cardiol. 2006;86(6):399-407. http://dx.doi.org/10.1590/S0066-782X2006000600001

6. Berry RB, Brooks R, Gamaldo CE, Harding SM, Lloyd RM, Marcus $\mathrm{CL}$, et al. The AASM Manual for the Scoring of Sleep and Associated Events: rules, terminology and technical specifications, version 2.2. Darien (IL): American Academy of Sleep Medicine; 2015

7. Pereira GDP, Ramos FA, Fomin DS, Alóe F, Pinto JA. Ronco e apneia-hipopneia obstrutiva do sono: indicações da somnoplastia e da uvulopalatofaringoplastia com laser de $\mathrm{CO}_{2}$. Int Arch Otorhinolaryngol. 2001;5(4)

8. Lacerda VMA, Cunha ACR, Souza IR, Vasconcelos RS, Melo LTM, Abdon APV. Qualidade de vida (QV) e aspectos clínicos de pacientes com síndrome da apneia obstrutiva do sono (SAOS) em tratamento com pressão positiva contínua nas vias aéreas (CPAP). Rev Bras Qual Vida. 2013;5(1):22-30. http://dx.doi.org/10.3895/S2175-08582013000100003

9. Ito FA, Ito RT, Moraes NM, Sakima T, Bezerra MLS, Meirelles RC Condutas terapêuticas para tratamento da síndrome da apnéia e hipopnéia obstrutiva do sono (SAHOS) e da síndrome da resistência das vias aéreas superiores (SRVAS) com enfoque no aparelho anti-ronco (AAR-ITO). Rev Dent Press Ortodon Ortop Facial. 2005;10(4):143-56.

http://dx.doi.org/10.1590/S1415-54192005000400015
10. Ciconelli RM, Ferraz MB, Santos W, Meinão I, Quaresma MR. Tradução para língua portuguesa e validação do questionário genérico de avaliação de qualidade de vida SF-36 (Brasil SF-36). Rev Bras Reumatol. 1999;39(3):143-50.

11. Bertolazi AN, Fagondes SC, Hoff LS, Pedro VD, Barreto SSM, Johns MW. Validação da escala de sonolência de Epworth em português para uso no Brasil. J Bras Pneumol. 2009;35(9):877-83.

http://dx.doi.org/10.1590/S1806-37132009000900009

12. Bixler EO, Vgontzas AN, Ten Have T, Tyson K, Kales A. Effects of age on sleep apnea in men: I. Prevalence and severity. Am $J$ Respir Crit Care Med. 1998;157(1):144-8. http://dx.doi.org/10.1164/ajrccm.157.1.9706079

13. Soares MC, de Azeredo Bittencourt LR, Zonato Al, Gregório LC. Application of the Kushida morphometric model in patients with sleep-disordered breathing. Braz $J$ Otorhinolaryngol. 2006;72(4):541-8

http://dx.doi.org/10.1590/S0034-72992006000400017

14. Pinto JA, Godoy LB, Marquis VW, Sonego TB, Leal C de F, Ártico MS. Medidas antropométricas preditoras da gravidade da apneia obstrutiva do sono. Braz J Otorhinolaryngol. 2011;77(4):516-21. http://dx.doi.org/10.1590/S1808-86942011000400017

15. Zancanella E, Haddad FM, Oliveira LA, Nakasato A, Duarte BB, Soares CF, et al. Obstructive sleep apnea and primary snoring: treatment. Braz J Otorhinolaryngol. 2014;80(1 Suppl 1):S17-28. http://dx.doi.org/10.5935/1808-8694.2014S002

16. Mediano O, Barceló A, de la Peña M, Gozal D, Agusti A, Barbé F. Hipersonolência diurna e variáveis polissonográficas em doentes com síndroma da apnéia do sono. Rev Port Pneumol. 2007;13(6):896-8.

http://dx.doi.org/10.1016/S0873-2159(15)30387-1

17. McArdle N, Kingshott R, Engleman HM, Mackay TW, Douglas NJ. Partners of patients with sleep apnoea/hypopnoea syndrome: effect of CPAP treatment on sleep quality and quality of life. Thorax. 2001;56(7):513-8.

http://dx.doi.org/10.1136/thorax.56.7.513

18. Silva RZM, Duarte RLM, Silveira FJM. Tratamento da apnéia obstrutiva do sono com pressão positiva contínua na via aérea. Pulmão RJ. 2010;19(3-4):83-7. 